\title{
An Integrated Management Supervisor for End-to-End Management of Heterogeneous Contents, Networks, and Terminals enabling Quality of Service
}

\author{
Christian Timmerer, Michael \\ Ransburg, Ingo Kofler, \\ Hermann Hellwagner \\ Klagenfurt University, Austria \\ Universitätsstrasse 65-67 \\ 9020 Klagenfurt \\ $+43 / 463 / 27003621$ \\ \{firstname.lastname\}@itec.uni- \\ klu.ac.at \\ Adam Lindsay, Michael Mackay \\ University of Lancaster, UK \\ Computing Department, InfoLab21, \\ Lancaster University, Lancaster, \\ LA1 4WA, UK \\ \{atl, m.mackay\} \\ @comp.lancs.ac.uk
}

\author{
Pedro Souto, Maria Andrade, \\ Pedro Carvalho, Hélder Castro \\ INESC, Porto, Portugal \\ Rua Dr. Roberto Frias 378 \\ P-4200-465 Porto \\ $+35 / 122 / 2094000$ \\ \{psouto, maria.andrade, \\ pedro.carvalho, \\ hcastro\}@inescporto.pt
Artur Lugmayr
NAMU Lab., Tampere Univ. of Technology, Finland
POB. 553, FIN-33101 Tampere +358/40/821 0558
lartur@acm.org

\author{
Mamadou Sidibé, Ahmed \\ Mehaoua, Li Fang \\ CNRS-PRISM, UVSQ, France \\ 45 avenue des Etats-Unis \\ 78035 Versailles \\ $+33 / 1 / 39254327$ \\ \{mas, mea, \\ li.fang\}@prism.uvsq.fr
}
Bernhard Feiten
T-Systems Enterprise Services, Germany
+493034972528
\{bernhard.feiten@t-
systems.com\}

\begin{abstract}
End-to-end support for Quality of Service (QoS) has been broadly discussed in the literature. Many technologies have been proposed, each focusing on specific aspects for providing QoS guarantees to the end user. However, the integrated management of the end-to-end chain preserving QoS in heterogeneous environments is still a significant issue and insufficiently addressed to date. In this paper we propose an integrated management supervisor that takes into account the requirements from all stakeholders along the multimedia content delivery chain and provides an end-to-end management solution enabling QoS to the end user. This architecture and the subsystems that can be distributed along the end-to-end chain are detailed in this paper.
\end{abstract}

\section{Categories and Subject Descriptors}

H.4.3 [Information Systems Applications]: Communications Applications.

\section{General Terms}

Management, Measurement, Performance, Design, Standardization.

\section{Keywords}

Integrated management, end-to-end, quality of service, multimedia, heterogeneity, MPEG-21

\section{INTRODUCTION}

Today there are many technologies in place which aim to enable end-to-end Quality of Service for advanced multimedia services [1]. However, for the most part, these technologies are neither integrated within an interoperable framework nor provide a means to effectively manage the end-to-end multimedia delivery chain. Thus, there exists a need for solutions based on an interoperable multimedia framework supporting the end-to-end management of heterogeneous contents, networks, and terminals while enabling Quality of Service (QoS) for the end user.

A framework that can be used for this purpose is the MPEG-21 multimedia framework, which enables the transparent and augmented use of multimedia resources across a wide range of networks, devices, user preferences, and communities [2]. In particular, MPEG-21 provides means for the transaction of Digital Items (i.e., multimedia resources and metadata within a standardized structure) among Users and whose functions can be categorized into six categories: declaration (and identification), digital rights management, adaptation, processing, systems, and miscellaneous aspects (i.e., reference software, conformance, etc.).

In this paper we describe an architecture for the integrated management of the end-to-end multimedia delivery chain that utilizes the MPEG-21 multimedia framework [3] and that enables QoS for the end-user, by adopting cross-layer techniques [4]. As this architecture has been developed in the course of the ENTHRONE II project [5] it is referred to as the ENTHRONE Integrated Management Supervisor (EIMS).

The remainder of this paper is organized as follows. The highlevel architecture of ENTHRONE for end-to-end management enabling QoS is described in Section 2. This section also highlights the cross-layer QoS adaptation concept. Section 3 describes EIMS with its functional building blocks and interfaces. Finally, Section 4 describes an example deployment of the architecture introduced here and Section 5 concludes the paper. 


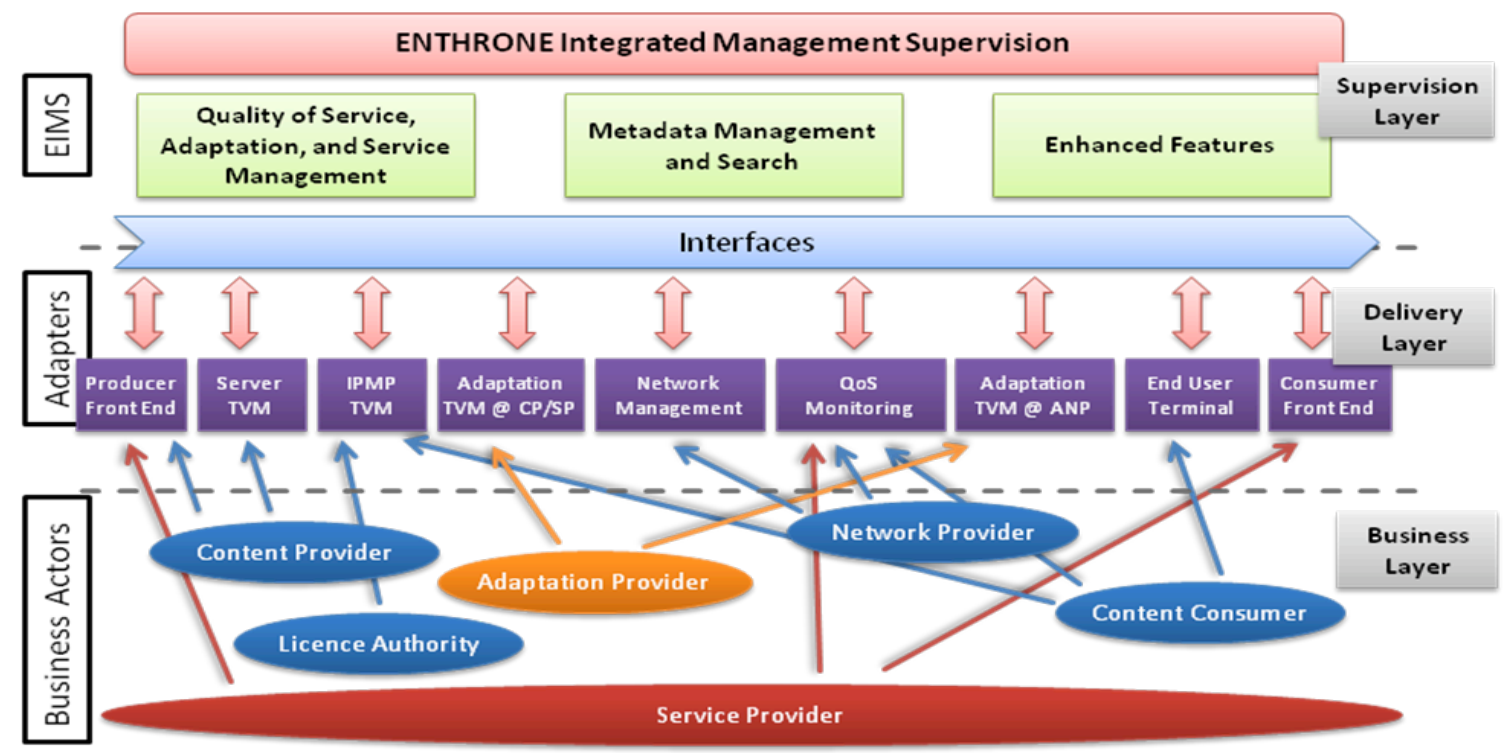

Figure 1 - ENTHRONE high-level architecture for end-to-end management enabling QoS.

\section{ENTHRONE OVERVIEW}

\subsection{End-to-End Management enabling QoS}

The ENTHRONE high-level architecture for end-to-end management enabling QoS is given in Figure 1. It comprises three layers:

The top layer is the supervision layer which role is to manage and to monitor the services that participate in the content delivery chain. It is implemented by the ENTHRONE Integrated Management Supervision (EIMS), which uses MPEG 21 interfaces to the components at the underlying service delivery layer. The EIMS is composed of several subsystems, that are put together to support specific use cases. These subsystems will be further explained in Section 3, and can be grouped in three main classes:

- QoS-based service management and multimedia content adaptation;

\section{- Metadata management and content search; and}

- Multicast, service monitoring, content caching, and content distribution networks, specified as enhanced features.

The delivery layer (middle) comprises all functionalities required for a proper content delivery with end-to-end management enabling QoS. An adapter is the implementation, in hardware or software, of some of this functionality. Figure 1 shows the main adapters, but is not intended to be exhaustive.

The business layer (bottom) specifies the concerned actors in the end-to-end QoS service chain. Each actor relies on the services of one or more components of the delivery layer. This paper focuses on the top layer and does not detail further the other two layers.

\subsection{Cross-layer QoS Adaptation Concept}

The ENTHRONE solution for QoS support is based on the concept of MPEG-21-enabled cross-layer adaptation. The idea behind this concept is to perform coordinated actions across several levels and layers along the end-to-end content delivery chain. The concept of MPEG-21-enabled cross-layer adaptation can be divided in the following three steps:

I. The ENTHRONE Cross-Layer Model (EXLM): The EXLM provides means for describing the relationship between QoS metrics at different levels - i.e., perceived QoS (PQoS), application QoS (AppQoS), and network QoS (NQoS) - and layers - i.e., according to the well-known ISO/OSI reference model - which may cooperate to improve the ability of applications to ensure certain objectives such as QoS guarantees, power savings, users preferences, etc.

II. The instantiation of the EXLM by utilizing MPEG-21 metadata: Description formats (i.e., tools) as specified within MPEG-21 Digital Item Adaptation (DIA) [6] are used to instantiate the EXLM for a specific use case scenario, e.g., Video-on-Demand. In particular, the Adaptation $Q o S$ (AQoS) description tool is used as the main component to describe the relationship between constraints, feasible adaptation operations satisfying these constraints, and associated utilities (qualities) [7]. The Usage Environment Description (UED) tools are used to describe the context information where Digital Items are consumed in terms of network conditions, terminal capabilities, user preferences, and conversion capabilities. Finally, the Universal Constraints Description (UCD) tools are used to express limitation and optimization constraints.

III. The Cross-Layer Adaptation Decision-Taking Engine (XLADTE): The XL-ADTE is part of an EIMS subsystem which provides the optimal parameter settings for media resource engines (e.g., TVMs) according to the EXLM by processing the metadata compliant to MPEG-21 DIA. In other words, the XL-ADTE is a generic (software) module that solves optimization problems [8] expressed by using MPEG-21 DIAbased metadata according to the EXLM. 


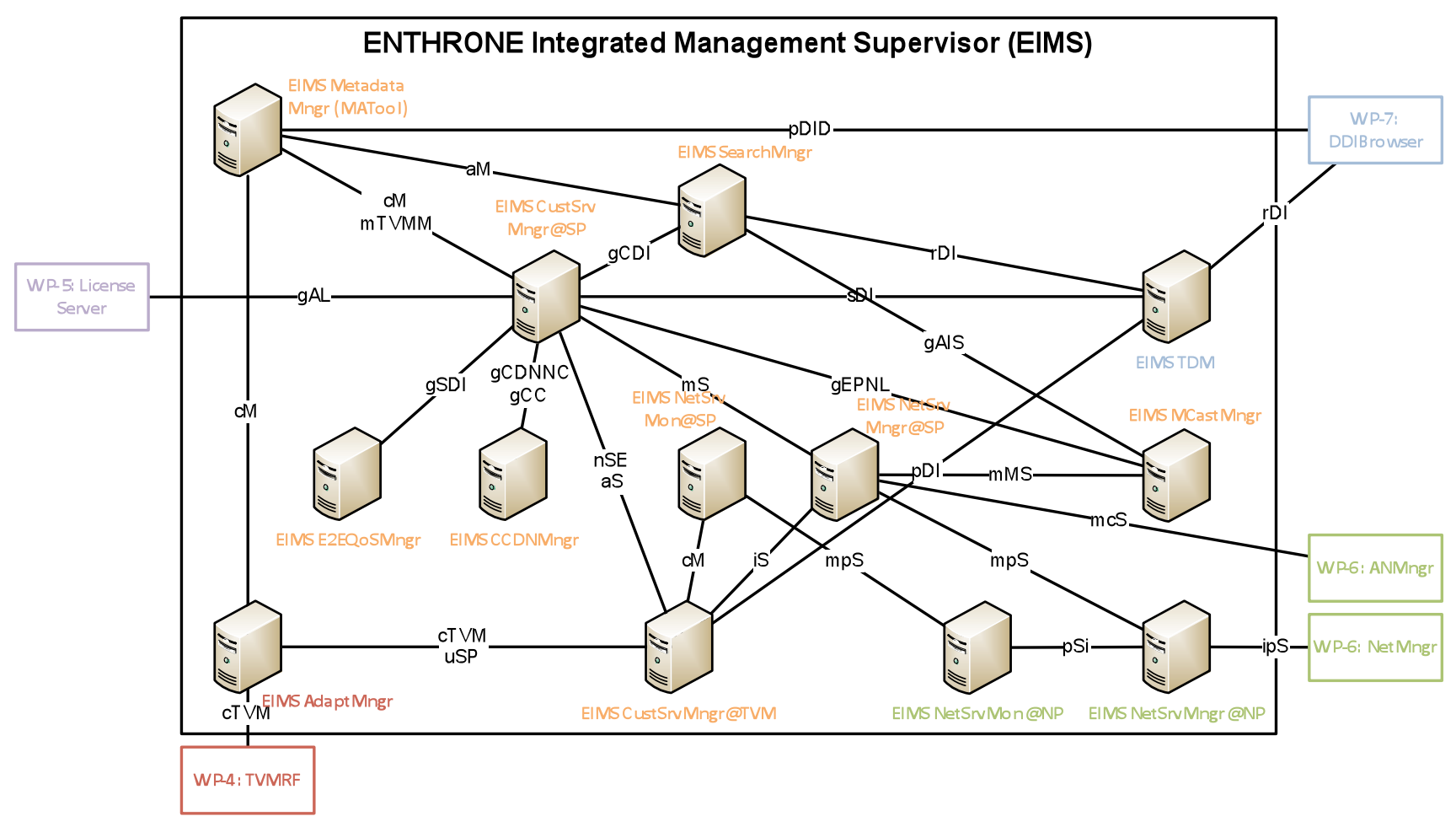

Figure 2 - Overview of the ENTHRONE Integrated Management Supervision (EIMS) architecture.

\section{ENTHRONE INTEGRATED MANAGEMENT SUPERVISOR (EIMS)}

\subsection{Architecture Overview}

The aim of the ENTHRONE Integrated Management Supervisor (EIMS) is to enable the deployment of multimedia services allowing for end-to-end management with QoS support across heterogeneous environments. Therefore, the EIMS provides a set of management subsystems, i.e., EIMS Managers, with predefined functionalities and interfaces - based on Web Services - which enable the construction of ENTHRONE-based services according to the requirements of various scenarios.

An overview of the architecture of the EIMS is depicted in Figure 2 highlighting the key EIMS Managers and its main interfaces. Note however that due to space constraints it is not possible to describe in detail every EIMS Manager and every interface shown in the figure and, thus, for further details the interested reader is referred to [9].

\subsection{End-to-End QoS Manager}

The aim of the End-to-End QoS Manager (E2E-QoS-Mngr) is to provide the best Digital Item configuration towards the content consumer taking into account various information coming from different business actors along the delivery chain. This information is ideally represented as MPEG-21 compliant metadata and encapsulated within the Digital Item Declaration which will be configured according to the requirements of the content consumer. The various metadata assets are briefly described in the following:

- The Digital Item Declaration (DID) comprising the content variations from which the E2E-QoS-Mngr may choose. The DID provides a representation of the Digital Item compliant to
MPEG-21 Part 2 [10] and is usually provided by the content provider (CP).

- The characteristics of the available television and multimedia (TVM) processors which may be selected by the E2E-QoS-Mngr based on the network characteristics or conditions (i.e., the information from the provider-to-provider Service Level Specification (pSLS)) between the content consumer and the various TVMs. The TVMs are described by a unified description model featuring MPEG-7 and MPEG-21 tools and is generally referred to as TVM Resource Function (TVMRF) [11].

- The capabilities and network information from the terminal of the content consumer including the class of service for the requested multimedia service. This kind of information is described in a format compliant to MPEG-21 Part 7 Usage Environment Description (UED) tools.

The E2E-QoS-Mngr implements a process of automated construction of a possibly suitable sequence of TVMs for a given set of multimedia content variations and a set of environmental requirements as described in [12].

The output of the E2E-QoS-Mngr is a DID configured with the chosen content variation including the location of the selected TVM and the corresponding QoS characteristics of the actual multimedia content.

\subsection{Service Manager}

The Service Manager (SrvMngr) is responsible for service management and can be further divided into four subsystems with distinguished functionalities: 
- The Customer Service Manager (CustSrvMngr) acts as a central component which provides the actual service towards the customer by implementing the service logic according to the service requirements. It instantiates other EIMS managers and coordinates the information flow within the EIMS.

- The Network Service Manager (NetSrvMngr) is responsible for managing network connectivity services used to transport the multimedia content with the requested QoS guarantees from its source to its consumers through several established pSLSs. Specifically, it encompasses the functionalities for service planning, provisioning, offering and fulfillment of the connectivity service, in a multi-domain context. The NetSrvMngr also strongly interacts with the Service Monitoring as described in the next bullet.

- Service Monitoring (ServMon) provides a means for monitoring the service with the aim to keep track of the endto-end QoS level of a particular service [13]. Service monitoring is mainly provided within the network on aggregated streams and within the CustSrvMngr for a particular service stream. Therefore, service monitoring provides means for mapping network QoS (NQoS) monitored on aggregated streams within the core network - to perceived QoS (PQoS) that is relevant on a per stream level [14].

- The Terminal Device Manager (TDM) enables the management of heterogeneous end-user devices in terms of capturing the capabilities of the terminal, PQoS probe configuration including handling its alarms, and license handling [15].

\subsection{Adaptation Manager}

The Adaptation Manager (AdaptMngr) aims to provide adaptation decisions according to dynamically varying context conditions coming from various sources across service and network layers. Thus, the adaptation manager hosts the Cross-Layer Adaptation Decision-Taking Engine (XL-ADTE) - see also Section 2.2 - and steers exactly one TVM (in a possible chain of TVMs). That is, the E2E-QoS-Mngr selects one (or more) TVM(s) during the service request and basically establishes the QoS-enabled end-toend multimedia service. The role of the AdaptMngr is actually to configure each TVM involved within the chain. Furthermore, it adjusts this configuration according to possible changing usage environment properties, dynamically received from ServMons and the TDM through the CustSrvMngr. Finally, it may provide updates for the various QoS probes along the multimedia service delivery chain.

\subsection{Metadata Manager}

The EIMS Metadata Manager is responsible for performing metadata related tasks within the scope of the EIMS. These tasks include:

- Aggregation and enrichment of metadata from different metadata sources;

- Contextual metadata collection from and retrieval by different EIMS components;

- Metadata conversion between different formats.

For further details of this EIMS subsystem the interested reader is referred to [16].

\subsection{Search Manager}

The Search Manager supports searching of Digital Items and browsing of DIDs in the DID database via a well-defined interface. The search and browsing is based on the data model specifically developed within ENTHRONE [17] and adopts the query format as defined by MPEG [18]. The data model supports high-level and low-level features associated to audio-visual content. That is, the Search Manager provides the following main functionalities:

- Search by making use of high-level features (e.g., keywords) as well as low-level features (e.g., color, shape, etc.);

- Relevance feedback by the user, through the submission of user's annotations upon browsing or consuming the content;

- Query by relevance feedback where feedback provided by users, who previously annotated Digital Items, is considered in the search process.

\subsection{Multicast Manager}

The Multicast Manager is responsible for all the tasks related to the management of multicast communication services in ENTHRONE. That is, the multicast overlay network and possibly the cross-layer multicast agent, which takes advantage of IP multicast in the last core network domain towards the customer [19]. The tasks that the Multicast Manager carries out can be grouped in two main categories:

- The overlay network configuration and administration, which includes the definition of so-called E-Nodes (i.e., a network component that is part of the multicast tree which performs packet forwarding and replication) as well as the subscription and invocation of contracts within network provides (pSLSs).

- The handling of multicast service requests, which includes the subscription and invocation of a multicast customer subscription (cSLA) in order to enable an end user to consume a particular QoS-enabled multicast stream.

\subsection{Caching and CDN Manager}

The Caching and CDN Manager (CCDNMngr) is the EIMS subsystem responsible for all the tasks related to supporting caching and CDNs in ENTHRONE. The aim of the CCDNMngr is to transparently supplement existing EIMS functionality by providing alternative content sources that will either improve the performance of the content streaming or improve the robustness and scalability of the architecture.

Within ENTHRONE, CDNs are considered to be a discrete entity holding complete, static copies of content and are managed either within a Service Provider (via the EIMS CCDNMngr) or externally by a separate entity. In contrast, Caching Nodes are managed as semi-autonomous standalone entities that dynamically cache portions of content. The main functionalities of the CCDNMngr include to:

- manage the provisioning of the content, i.e., inject content into the $\mathrm{CDN}$ or upload content to the local caches;

- manage the placement of content in different CDN Nodes including collecting statistics used as input to the content placement algorithms; 


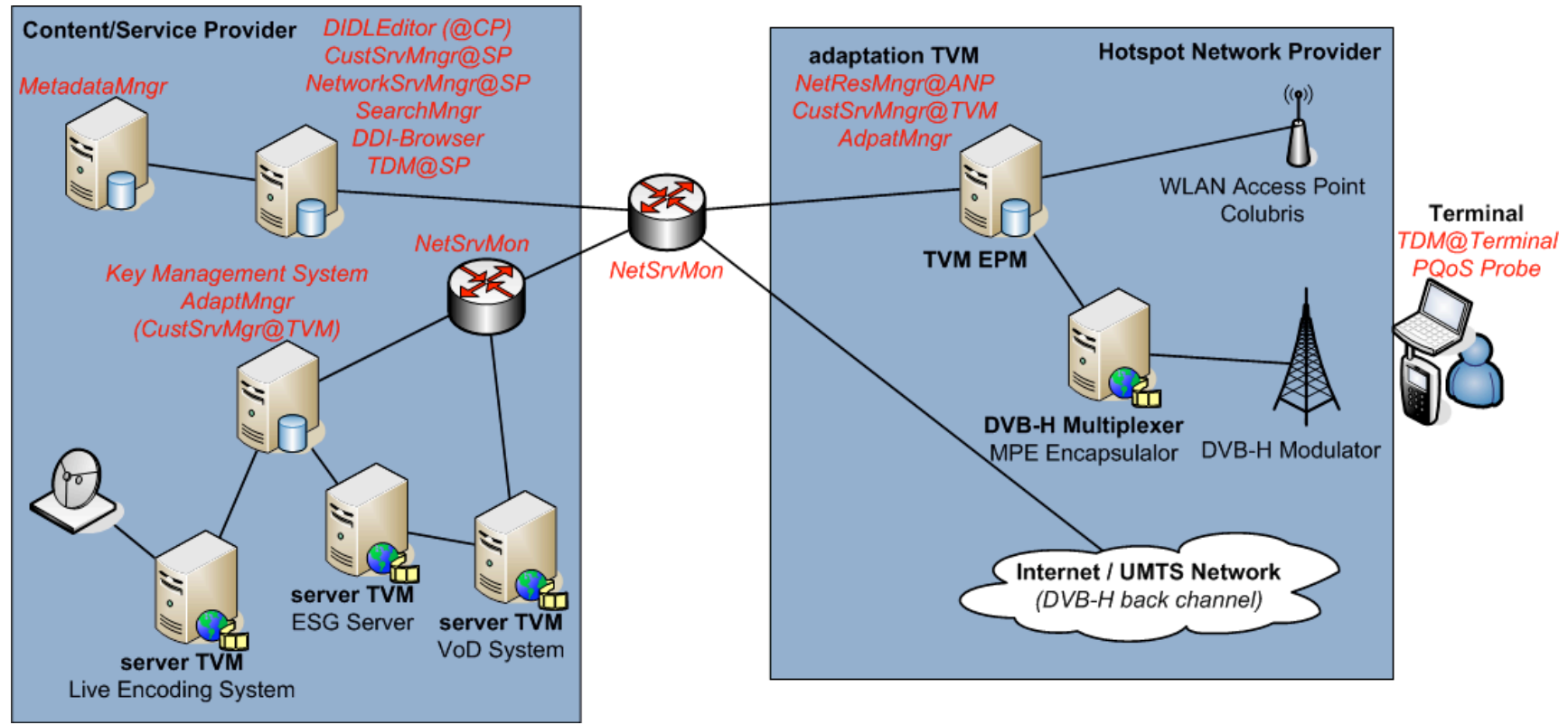

Figure 3 - EIMS Deployment Architecture.

- manage the cache policy which tunes the performance of Caching Nodes (caching method, replacement policy, etc) based on content usage statistics; and

- select a Caching Node and/or CDN Node to stream/deliver content in response to a consumer request, taking into account different factors such as the consumer location and the state of the nodes.

\section{EIMS DEPLOYMENT EXAMPLE}

\subsection{Introduction}

This section provides a brief description of a deployment scenario of the EIMS as part of the German Pilot Island (GPI) documented in [20] and to be demonstrated at IFA 2008 [21]. The German pilot aims to develop several mobile multimedia service scenarios introduced in Section 4.2. The actual deployment architecture is highlighted in Section 4.3.

\subsection{Mobile Service Scenarios}

The GPI demonstrates how the ENTHRONE systems can manage the content provisioning and the play-out of audiovisual streams in a heterogeneous network landscape. Therefore, it establishes three mobile services:

- VideoLogChannel for consuming both professional and usergenerated content on the go via various channels (e.g., WLAN, DVB-H).

- SoccerChannel offering protected live soccer games all the time prior to subscription via a Web interface. The service is offered via traditional IP-based channels but also over DVBH.

- The MultiRadio service is an interactive enhanced radio service where users are able to "watch" additional information (e.g., pictures, slideshows, or videos) while listening to a live radio program.

\subsection{Deployment Architecture}

The possible architecture for a deployment of the EIMS enabling the services as described in the previous section is depicted in Figure 3. In the following, a brief walkthrough is provided.

The content provider authors the Digital Items using the DIDLEditor, a Web-based editing tool, and uploads its declaration to the MetadataMngr hosted by the service provider. The actual multimedia assets are provided through various server TVMs depending on the delivery channel (i.e., TVM On-Demand, TVM Broadcast-DVB-H/-IP). The hotspot network operator maintains an error protection module (TVM EPM) in order to cope with varying conditions in the access network. Note that all TVMs are controlled by the appropriate EIMS subsystems (CustSrvMngr@TVM and AdaptMngr) through the TVMRF interface.

The terminal accesses the Digital Items through a Web interface by utilizing the TDM. First, the user may search for Digital Items which is accomplished by the SearchMngr that interacts with the MetadataMngr. Second, the CustSrvMngr@SP delegates the result of this search to the E2EQoSMngr which provides the best configuration among the available content variations and according to the terminal/network capabilities. The terminal capabilities are provided during the service request and the NetSrvMngr@SP (which is connected with the NetResMngr@ANP) provides the current network conditions. Third, the network is configured according to the selected Digital Item provided by the E2EQoSMngr and, subsequently, the TVMs are configured based on this selection also.

In the case of a quality degradation during service delivery we are able to react thanks to the service monitoring deployed in the network (i.e., NetServMon) and on the terminal (i.e., PQoS Probe). The monitoring information is reported to the CustSrvMngr@TVM which instructs the AdaptMngr to reconfigure the TVMs - possibly both at the service provider and hotspot network operator - in order keep the service level for which the customer has subscribed. 
For further details about this deployment the interested user is referred to [20].

\section{CONCLUSIONS}

In this paper we have introduced an integrated management architecture for end-to-end management of the entire audio/visual delivery chain with QoS enabled. This is mainly achieved by adopting the MPEG-21 multimedia framework in combination with cross-layer adaptation techniques. The overall architecture has been described briefly and the main functional units (i.e., EIMS managers) as well as its interfaces have been introduced. Finally, an example deployment of the architecture has been described. Other deployments exist in other countries [20], e.g. a pilot in Lancaster uses CDNs to provide services to a university and to local communities. We expect these pilots to validate the feasibility of the EIMS in particular and of the ENTHRONE approach in general.

\section{ACKNOWLEDGMENTS}

This work is supported by the European Commission in the context of the ENTHRONE project (IST-1-507637). Further information is available at http://www.ist-enthrone.org.

\section{REFERENCES}

[1] T. Guenkova-Luy, A. Kassler, D. Mandato, "End-to-End Quality of Service Coordination for Mobile Multimedia Applications", IEEE Journal on Selected Areas in Communications, vol. 22, no. 5, Jun. 2004.

[2] F. Pereira, J.R. Smith, A. Vetro, (eds.), "Special Section on MPEG-21", IEEE Trans. on Multimedia, vol. 7, no. 3, Jun. 2005.

[3] I. Burnett, F. Pereira, R. Van de Walle, R. Koenen (eds.), The MPEG-21 Book, Wiley, 2006.

[4] I. Kofler, C. Timmerer, H. Hellwagner, T. Ahmed, "Towards MPEG-21-based Cross-layer Multimedia Content Adaptation", Proc. 2nd Int'l Workshop on Semantic Media Adaptation and Personalization (SMAP 2007), London, UK, Dec. 2007.

[5] O. Negru, "End-to-End QoS through Integrated Management of Content, Networks and Terminals: The ENTHRONE Project", Proc. 1st Int'l Workshop on Wireless Internet Services (WISe 2008), Doha, Qatar, Apr. 2008.

[6] A. Vetro, C. Timmerer, "Digital Item Adaptation: Overview of Standardization and Research Activities", IEEE Trans. on Multimedia, vol. 7, no. 3, pp. 418-426, Jun. 2005.

[7] D. Mukherjee, E. Delfosse, J.-G. Kim, Y. Wang, "Optimal Adaptation Decision-Taking for Terminal and Network Quality-of-Service", IEEE Trans. on Multimedia, vol. 7, no. 3, pp. 454-462, Jun. 2005.

[8] I. Kofler, C. Timmerer, H. Hellwagner, A. Hutter, F. Sanahuja, "Efficient MPEG-21-based Adaptation DecisionTaking for Scalable Multimedia Content", Proc. 14th SPIE
Annual Electronic Imaging Conf. - Multimedia Computing and Networking (MMCN 2007), San Jose, CA, USA, Jan./Feb. 2007.

[9] ENTHRONE, http://ist-enthrone.org/.

[10] I.S. Burnett, S.J. Davis, G.M. Drury, "MPEG-21 Digital Item Declaration and Identification - Principles and Compression", IEEE Trans. on Multimedia, vol. 7, no. 3, pp. 400-407, Jun. 2005.

[11] A. Chernilov, I. Arbel, "Content Handling and Content Protection under E2E QoS Distribution", Proc. Int'l Conf. on Automation, Quality and Testing, Robotics (AQTR 2008), Cluj-Napoca, Romania, May 2008.

[12] D. Jannach, K. Leopold, C. Timmerer, H. Hellwagner, "A Knowledge-based Framework for Multimedia Adaptation", Applied Intelligence, vol. 24, no. 2, pp. 109-125, Apr. 2006.

[13] M. Sidibé, A. Mehaoua "Service Monitoring System for Dynamic Service Adaptation in Multi-domain and Heterogeneous Networks", Proc. 9th Int'l Workshop on Image Analysis for Multimedia Interactive Services (WIAMIS 2008), Klagenfurt, Austria, May 2008.

[14] H. Koumaras, A. Kourtis, C.-H. Lin, C.-K. Shieh, "A Theoretical Framework for End-to-End Video Quality Prediction of MPEG-based Sequences", Proc. 3rd Int'l Conf. on Networking and Services (ICNS 2007), Athens, Greece, Jun. 2007.

[15] B. Shao, D. Renzi, M. Mattavelli, S. Battista, S. Keller, "A Multimedia Terminal Supporting Adaptation for QoS Control", Proc. 9th Int'l Workshop on Image Analysis for Multimedia Interactive Services (WIAMIS 2008), Klagenfurt, Austria, May 2008.

[16] A. Lugmayr, "The ENTHRONE 2 Metadata Management Tool (MATool)", Proc. 1st Int'l Workshop on Wireless Internet Services (WISe 2008), Doha, Qatar, Apr. 2008.

[17] P. Carvalho, M. T. Andrade, C. Alberti, H. Castro, C. Calistru, P. de Cuetos, "A unified data model and system support for the context-aware access to multimedia content", Proc. Int'l Workshop on Multimedia Semantics - The role of metadata", Aachen, Germany, Mar. 2007.

[18] M. Gruhne, R. Tous, M. Döller, J. Delgado, H. Kosch, "MP7QF: An MPEG-7 Query Format", Proc. 3rd Int'l Conf. on Automated Production of Cross Media Content for Multichannel Distribution (AXMEDIS 2007), Barcelona, Spain, Nov. 2007.

[19] A. Mehaoua, L. Fang, "Multicast Provisioning and Multicast Service Management", ENTHRONE Workshop at WIAMIS 2008, Klagenfurt, Austria, May 2008.

[20] T. Ahmed (ed.), "Pilot and services integration and tests", ENTHRONE II Deliverable D27, Feb. 2008.

[21] Internationale Funkausstellung (IFA), Aug. 2008, last accessed: Jun. 2008. 